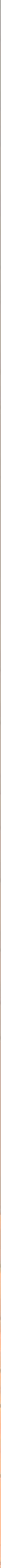




\title{
Intermediate co-t-structures, two-term silting objects, $\tau$-tilting modules, and torsion classes
}

\author{
Osamu lyama, Peter Jørgensen and Dong Yang
}

If $(A, B)$ and $\left(A^{\prime}, B^{\prime}\right)$ are co- $t$-structures of a triangulated category, then $\left(A^{\prime}, B^{\prime}\right)$ is called intermediate if $A \subseteq A^{\prime} \subseteq \Sigma A$. Our main results show that intermediate co- $t$-structures are in bijection with two-term silting subcategories, and also with support $\tau$-tilting subcategories under some assumptions. We also show that support $\tau$-tilting subcategories are in bijection with certain finitely generated torsion classes. These results generalise work by Adachi, Iyama, and Reiten.

\section{Introduction}

The aim of this paper is to discuss the relationship between the following objects:

- Intermediate co- $t$-structures.

- Two-term silting subcategories.

- Support $\tau$-tilting subcategories.

- Torsion classes.

The motivation is that if $T$ is a triangulated category with suspension functor $\Sigma$ and $(\mathrm{X}, \mathrm{Y})$ is a $t$-structure of $\mathrm{T}$ with heart $\mathrm{H}=\mathrm{X} \cap \Sigma \mathrm{Y}$, then there is a bijection between "intermediate" $t$-structures $\left(\mathrm{X}^{\prime}, \mathrm{Y}^{\prime}\right)$ with $\Sigma \mathrm{X} \subseteq \mathrm{X}^{\prime} \subseteq \mathrm{X}$ and torsion pairs of $H$. This is due to [Beligiannis and Reiten 2007, Theorem 3.1] and [Happel et al. 1996, Proposition 2.1]; see [Woolf 2010, Proposition 2.3].

We will study a co- $t$-structure analogue of this which also involves silting subcategories, that is, full subcategories $S \subseteq T$ with thick closure equal to $T$ which satisfy $\operatorname{Hom}_{\mathrm{T}}\left(\mathrm{S}, \Sigma^{i} \mathrm{~S}\right)=0$ for $i \geq 1$. Silting subcategories are a useful generalisation of tilting subcategories.

The next theorem follows from the bijection between bounded co- $t$-structures and silting subcategories in [Mendoza Hernández et al. 2013, Corollary 5.9]. See [Pauksztello 2008] and [Aihara and Iyama 2012] for background on co- $t$-structures

MSC2010: primary 18E30; secondary 18E40.

Keywords: co- $t$-structures, two-term silting objects, $\tau$-tilting modules, torsion classes. 
and silting subcategories. Note that the co-heart of a co- $t$-structure $(A, B)$ is $A \cap \Sigma^{-1} B$. If $F, G$ are full subcategories of a triangulated category, then $F * G$ denotes the full subcategory of objects $e$ which permit a distinguished triangle $f \rightarrow e \rightarrow g$ with $f \in \mathrm{F}, g \in \mathrm{G}$.

Theorem 0.1 (Theorem 2.2). Let $\mathrm{T}$ be a triangulated category, (A, B) a bounded co$t$-structure of $\mathrm{T}$ with co-heart $\mathrm{S}$. Then we have a bijection between the following sets:

(i) Co-t-structures $\left(\mathrm{A}^{\prime}, \mathrm{B}^{\prime}\right)$ of $\mathrm{T}$ with $\mathrm{A} \subseteq \mathrm{A}^{\prime} \subseteq \Sigma \mathrm{A}$.

(ii) Silting subcategories of $\mathrm{T}$ which are in $\mathrm{S} * \Sigma \mathrm{S}$.

The co- $t$-structures in (i) are called intermediate. The silting subcategories in (ii) are called two-term, motivated by the existence of a distinguished triangle $s_{1} \rightarrow s_{0} \rightarrow s^{\prime}$ with $s_{i} \in \mathrm{S}$ for each $s^{\prime} \in \mathrm{S}^{\prime}$. The theorem reduces the study of intermediate co-t-structures to the study of two-term silting subcategories.

Our main results on two-term silting subcategories and $\tau$-tilting theory can be summed up as follows. We extend the notion of support $\tau$-tilting modules for finitedimensional algebras over fields given in [Adachi et al. 2014] to essentially small additive categories; see Definitions 1.3 and 1.5. For a commutative ring $\mathbb{k}$, we say that a $\mathbb{k}$-linear category is Hom-finite if each Hom-set is a finitely generated $\mathbb{k}$-module.

Theorem 0.2 (Theorems 3.4 and 4.6). Let $\mathrm{T}$ be a triangulated category with a silting subcategory $\mathrm{S}$. Assume that each object of $\mathrm{S} * \Sigma \mathrm{S}$ can be written as a direct sum of indecomposable objects unique up to isomorphism. Then there is a bijection between the following sets:

(i) Silting subcategories of $\mathrm{T}$ which are in $\mathrm{S} * \Sigma \mathrm{S}$.

(ii) Support $\tau$-tilting pairs of $\bmod \mathrm{S}$.

If $\mathrm{T}$ is Krull-Schmidt, $\mathbb{k}$-linear and Hom-finite over a commutative ring $\mathbb{k}$, and $\mathrm{S}=$ add $s$ for a silting object $s$, then there is a bijection between the following sets:

(iii) Basic silting objects of $\mathrm{T}$ which are in $\mathrm{S} * \Sigma \mathrm{S}$, modulo isomorphism.

(iv) Basic support $\tau$-tilting modules of $\bmod E$, modulo isomorphism, where $E=$ $\operatorname{End}_{\mathrm{T}}(s)$.

Note that in this case, there is a bijection between (i) and (iii) by [Aihara and Iyama 2012, Proposition 2.20, Lemma 2.22(a)].

Note that Theorem 0.2 is a much stronger version of Theorem 3.2 of [Adachi et al. 2014], where T is assumed to be the homotopy category of bounded complexes of finitely generated projective modules over a finite-dimensional algebra $\Lambda$ over a field, and $s$ is assumed to be $\Lambda$. 
Moreover, we give the following link between $\tau$-tilting theory and torsion classes. Our main result shows that support $\tau$-tilting pairs correspond bijectively with certain finitely generated torsion classes, which is a stronger version of [Adachi et al. 2014, Theorem 2.7]. Note that Fac $M$ is the subcategory of Mod $C$ consisting of factor objects of finite direct sums of objects of $M$, and $P(T)$ denotes the Ext-projective objects of T; see Definition 1.7.

Theorem 0.3 (Theorem 5.1). Let $\mathbb{k}$ be a commutative noetherian local ring and $\mathrm{C}$ an essentially small, Krull-Schmidt, $\mathbb{k}$-linear Hom-finite category. There is a bijection $\mathrm{M} \mapsto \mathrm{Fac} \mathrm{M}$ from the first of the following sets to the second:

(i) Support $\tau$-tilting pairs (M, E) of $\bmod C$.

(ii) Finitely generated torsion classes $\mathrm{T}$ of $\mathrm{Mod} \mathrm{C}$ such that each finitely generated projective $\mathrm{C}$-module has a left $\mathrm{P}(\mathrm{T})$-approximation.

\section{Basic definitions}

Let $\mathrm{C}$ be an additive category. When we say that $\mathrm{U}$ is a subcategory of $\mathrm{C}$, we always assume $\mathrm{U}$ is full and closed under finite direct sums and direct summands. For a collection $\mathrm{U}$ of objects of $\mathrm{C}$, we denote by add $\mathrm{U}$ the smallest subcategory of $\mathrm{C}$ containing $\mathrm{U}$.

Let $C$ be an essentially small additive category. We write Mod $C$ for the abelian category of contravariant additive functors from $\mathrm{C}$ to the category of abelian groups, and $\bmod C$ for the full subcategory of finitely presented functors; see [Auslander 1974, pp. 184, 204].

The suspension functor of a triangulated category is denoted by $\Sigma$.

We first recall the notions of co- $t$-structures and silting subcategories.

Definition 1.1. Let $T$ be a triangulated category. A co-t-structure on $T$ is a pair $(A, B)$ of full subcategories of $T$ such that:

(i) $\Sigma^{-1} \mathrm{~A} \subseteq \mathrm{A}$ and $\Sigma \mathrm{B} \subseteq \mathrm{B}$.

(ii) $\operatorname{Hom}_{\mathrm{T}}(a, b)=0$ for $a \in \mathrm{A}$ and $b \in \mathrm{B}$.

(iii) For each $t \in \mathrm{T}$ there is a triangle $a \rightarrow t \rightarrow b \rightarrow \Sigma a$ in $\mathrm{T}$ with $a \in \mathrm{A}$ and $b \in \mathrm{B}$. The co-heart is defined as the intersection $A \cap \Sigma^{-1} B$. See [Pauksztello 2008; Bondarko 2010].

Definition 1.2. Let $T$ be a triangulated category.

(i) A subcategory $\mathrm{U}$ of $\mathrm{T}$ is called a presilting subcategory if $\mathrm{T}\left(u, \Sigma^{\geq 1} u^{\prime}\right)=0$ for any $u, u^{\prime} \in \mathrm{U}$.

(ii) A presilting subcategory $\mathrm{S} \subseteq \mathrm{T}$ is a silting subcategory if thick $(\mathrm{S})=\mathrm{T}$; see [Aihara and Iyama 2012, Definition 2.1(a)]. Here thick(S) denotes the smallest thick subcategory of $\mathrm{T}$ containing $\mathrm{S}$. 
(iii) An object $u \in \mathrm{T}$ is called a presilting object if it satisfies $\mathrm{T}\left(u, \Sigma^{\geq 1} u\right)=0$, namely, if $\operatorname{add}(u)$ is a presilting subcategory. Similarly an object $u \in \mathrm{T}$ is called a silting object if $\operatorname{add}(u)$ is a silting subcategory.

Next we introduce the notion of support $\tau$-tilting subcategories.

Definition 1.3. Let $C$ be an essentially small additive category.

(i) Let $\mathrm{M}$ be a subcategory of $\bmod \mathrm{C}$. A class $\left\{P_{1} \stackrel{\pi^{m}}{\rightarrow} P_{0} \rightarrow m \rightarrow 0 \mid m \in \mathrm{M}\right\}$ of projective presentations in $\bmod C$ is said to have property $(S)$ if

$$
\operatorname{Hom}_{\bmod C}\left(\pi^{m}, m^{\prime}\right): \operatorname{Hom}_{\bmod C}\left(P_{0}, m^{\prime}\right) \rightarrow \operatorname{Hom}_{\bmod C}\left(P_{1}, m^{\prime}\right)
$$

is surjective for any $m, m^{\prime} \in \mathrm{M}$.

(ii) A subcategory $\mathrm{M}$ of $\bmod \mathrm{C}$ is said to be $\tau$-rigid if there is a class of projective presentations $\left\{P_{1} \rightarrow P_{0} \rightarrow m \rightarrow 0 \mid m \in \mathrm{M}\right\}$ which has property (S).

(iii) A $\tau$-rigid pair of $\bmod C$ is a pair $(M, E)$, where $M$ is a $\tau$-rigid subcategory of $\bmod C$ and $\mathrm{E} \subseteq \mathrm{C}$ is a subcategory with $\mathrm{M}(\mathrm{E})=0$, that is, $m(e)=0$ for each $m \in \mathrm{M}$ and $e \in \mathrm{E}$.

(iv) A $\tau$-rigid pair (M, E) is support $\tau$-tilting if $\mathrm{E}=\operatorname{Ker}(\mathrm{M})$ and for each $s \in \mathrm{C}$ there exists an exact sequence $\mathrm{C}(-, s) \stackrel{f}{\longrightarrow} m^{0} \rightarrow m^{1} \rightarrow 0$ with $m^{0}, m^{1} \in \mathrm{M}$ such that $f$ is a left $\mathrm{M}$-approximation.

It is useful to recall the notion of Krull-Schmidt categories:

Definition 1.4. An additive category $C$ is called Krull-Schmidt if each of its objects is the direct sum of finitely many objects with local endomorphism rings. It follows that these finitely many objects are indecomposable and determined up to isomorphism; see [Bass 1968, Theorem I.3.6]. It also follows that $\mathrm{C}$ is idempotent complete; that is, for an object $c$ of $\mathrm{C}$ and an idempotent $e \in \mathrm{C}(c, c)$, there exist objects $c_{1}$ and $c_{2}$ such that $c=c_{1} \oplus c_{2}$ and $e=\mathrm{id}_{c_{1}}$; see [Keller 2013, 5.1].

(i) An object $c \in \mathrm{C}$ is basic if it has no repeated indecomposable direct summands.

(ii) For an object $c \in \mathrm{C}$, let $\#_{\mathrm{C}}(c)$ denote the number of pairwise nonisomorphic indecomposable direct summands of $c$.

The following is a version of Definition 1.3 for rings:

Definition 1.5. Let $E$ be a ring such that $\bmod E$ is Krull-Schmidt.

(i) A module $U \in \bmod E$ is called $\tau$-rigid if there is a projective presentation $P_{1} \stackrel{\pi}{\longrightarrow} P_{0} \rightarrow U \rightarrow 0$ in $\bmod E$ such that $\operatorname{Hom}_{E}(\pi, U)$ is surjective.

(ii) A $\tau$-rigid module $U \in \bmod E$ is called support $\tau$-tilting if there is an idempotent $e \in E$ which satisfies $U e=0$ and $\#_{\bmod E}(U)=\#_{\operatorname{prj}(E / E e E)}(E / E e E)$. 
Remark 1.6. Part (ii) of the definition makes sense because $\operatorname{prj}(E / E e E)$ is KrullSchmidt. Namely, since $\bmod E$ is Krull-Schmidt, it follows that prj $E$ is KrullSchmidt with additive generator $E_{E}$. The same is hence true for $(\operatorname{prj} E) /[\operatorname{add} e E]$ for each idempotent $e \in E$, and it is not hard to check that the endomorphism ring of $E_{E}$ in $(\operatorname{prj} E) /[$ add $e E]$ is $E / E e E$, so there is an equivalence of categories

$$
(\operatorname{prj} E) /[\operatorname{add} e E] \stackrel{\sim}{\rightarrow} \operatorname{prj}(E / E e E) .
$$

Hence $\operatorname{prj}(E / E e E)$ is Krull-Schmidt.

If $E$ is a finite-dimensional algebra over a field, then the definition coincides with the original definition of basic support $\tau$-tilting modules by Adachi, Iyama and Reiten [Adachi et al. 2014, Definition 0.1(c)].

Finally we introduce the notion of torsion classes:

Definition 1.7. Let $C$ be an essentially small additive category and $T$ a full subcategory of Mod C.

(i) We say that $\mathrm{T}$ is a torsion class if it is closed under factor modules and extensions.

(ii) For a subcategory M of Mod C, we denote by Fac M the subcategory of Mod C consisting of factor objects of objects of $M$.

(iii) We say that a torsion class $\mathrm{T}$ is finitely generated if there exists a full subcategory $\mathrm{M}$ of mod $\mathrm{C}$ such that $\mathrm{T}=\mathrm{Fac} \mathrm{M}$. Clearly the objects in Fac $\mathrm{M}$ are finitely generated C-modules, which are not necessarily finitely presented.

(iv) An object $t$ of a torsion class $\mathrm{T}$ is Ext-projective if $\operatorname{Ext}_{\text {Mod } \mathrm{C}}^{1}(t, \mathrm{~T})=0$. We denote by $P(T)$ the full subcategory of $T$ consisting of all Ext-projective objects of $T$.

\section{Silting subcategories and co- $t$-structures}

In this section, $T$ is an essentially small, idempotent complete triangulated category. Let $(A, B)$ be a co- $t$-structure on $T$. It follows from the definition that

$$
\begin{aligned}
& \mathrm{A}=\{t \in \mathrm{T} \mid \operatorname{Hom}(t, b)=0 \text { for all } b \in \mathrm{B}\}, \\
& \mathrm{B}=\{t \in \mathrm{T} \mid \operatorname{Hom}(a, t)=0 \text { for all } a \in \mathrm{A}\} .
\end{aligned}
$$

In particular, both $A$ and $B$ are idempotent complete and extension closed. Hence so is the co-heart $S=A \cap \Sigma^{-1} B$. Set

$\mathrm{S} * \Sigma \mathrm{S}=\left\{t \in \mathrm{T} \mid\right.$ there is a triangle $s_{1} \rightarrow s_{0} \rightarrow t \rightarrow \Sigma s_{1}$ with $\left.s_{0}, s_{1} \in \mathrm{S}\right\} \subseteq \mathrm{T}$.

The following lemma will often be used without further remark: 
Lemma 2.1. There is an equality $\mathrm{S} * \Sigma \mathrm{S}=\Sigma \mathrm{A} \cap \Sigma^{-1} \mathrm{~B}$. As a consequence, $\mathrm{S} * \Sigma \mathrm{S}$ is idempotent complete and extension closed.

Proof. The inclusion $\mathrm{S} * \Sigma \mathrm{S} \subseteq \Sigma \mathrm{A} \cap \Sigma^{-1} \mathrm{~B}$ is clear, because both $\mathrm{S}$ and $\Sigma \mathrm{S}$ are contained in $\Sigma A \cap \Sigma^{-1} B$, which is extension closed. Next we show the opposite inclusion. Let $t \in \Sigma \mathrm{A} \cap \Sigma^{-1} \mathrm{~B}$. Then by Definition 1.1(iii) there is a triangle $a \rightarrow t \rightarrow b \rightarrow \Sigma a$ with $a \in \mathrm{A}$ and $b \in \mathrm{B}$. Since both $t$ and $\Sigma a$ are in $\Sigma \mathrm{A}$, so is $b$ due to the fact that $\mathrm{A}$ is extension closed. Thus $b \in \Sigma \mathrm{A} \cap \mathrm{B}=\Sigma \mathrm{S}$. Similarly, one shows that $a \in \mathrm{S}$. Thus we obtain a triangle $\Sigma^{-1} b \rightarrow a \rightarrow t \rightarrow b$ with $\Sigma^{-1} b$ and $a$ in $\mathrm{S}$, meaning that $t \in \mathrm{S} * \Sigma \mathrm{S}$.

It is easy to see that $\operatorname{Hom}\left(s, \Sigma^{\geq 1} s^{\prime}\right)=0$ for any $s, s^{\prime} \in \mathrm{S}$. That is, $\mathrm{S}$ is a presilting subcategory of $\mathrm{T}$. The co- $t$-structure $(\mathrm{A}, \mathrm{B})$ is said to be bounded if

$$
\bigcup_{n \in \mathbb{Z}} \Sigma^{n} \mathrm{~B}=\mathrm{T}=\bigcup_{n \in \mathbb{Z}} \Sigma^{n} \mathrm{~A}
$$

Theorem 2.2 [Mendoza Hernández et al. 2013, Corollary 5.9]. There is a bijection $(A, B) \mapsto A \cap \Sigma^{-1} B$ from the first of the following sets to the second:

(i) Bounded co-t-structures on T.

(ii) Silting subcategories of $\mathrm{T}$.

This result has the following consequence:

Theorem 2.3. Let $(\mathrm{A}, \mathrm{B})$ be a bounded co-t-structure on $\mathrm{T}$ with co-heart $\mathrm{S}$. Then there is a bijection $\left(\mathrm{A}^{\prime}, \mathrm{B}^{\prime}\right) \mapsto \mathrm{A}^{\prime} \cap \Sigma^{-1} \mathrm{~B}^{\prime}$ from the first of the following sets to the second:

(i) Bounded co-t-structures $\left(\mathrm{A}^{\prime}, \mathrm{B}^{\prime}\right)$ on $\mathrm{T}$ with $\mathrm{A} \subseteq \mathrm{A}^{\prime} \subseteq \Sigma \mathrm{A}$.

(ii) Silting subcategories of $\mathrm{T}$ which are in $\mathrm{S} * \Sigma \mathrm{S}$.

Proof. Let $\left(\mathrm{A}^{\prime}, \mathrm{B}^{\prime}\right)$ be a bounded co-t $t$-structure on $\mathrm{T}$ with $\mathrm{A} \subseteq \mathrm{A}^{\prime} \subseteq \Sigma \mathrm{A}$. Then $\mathrm{B} \supseteq \mathrm{B}^{\prime} \supseteq \Sigma \mathrm{B}$. It follows that $\mathrm{A}^{\prime} \cap \Sigma^{-1} \mathrm{~B}^{\prime} \subseteq \Sigma \mathrm{A} \cap \Sigma^{-1} \mathrm{~B}=\mathrm{S} * \Sigma \mathrm{S}$. The last equality is by Lemma 2.1 .

Let $\mathrm{S}^{\prime}$ be a silting subcategory of $\mathrm{T}$ which is in $\mathrm{S} * \Sigma \mathrm{S}$. Let $\mathrm{A}^{\prime}$ be the smallest extension closed subcategory of $T$ containing $\Sigma^{\leq 0} S^{\prime}$ and $B^{\prime}$ the smallest extension closed subcategory of $T$ containing $\Sigma^{\geq 1} \mathrm{~S}^{\prime}$. Then $\left(\mathrm{A}^{\prime}, \mathrm{B}^{\prime}\right)$ is the bounded co- $t$ structure corresponding to $S^{\prime}$ as in Theorem 2.2; see [Mendoza Hernández et al. 2013, Corollary 5.9]. Since $S^{\prime} \subseteq S * \Sigma S$, it follows that $A^{\prime}$ is contained in the smallest extension closed subcategory of $\mathrm{T}$ containing $\Sigma^{\leq 1} \mathrm{~S}$, which is exactly $\Sigma A$. Similarly, one shows that $B^{\prime}$ is contained in $B$, implying that $A^{\prime}$ contains $A$. Thus, $A \subseteq A^{\prime} \subseteq \Sigma A$. 
The co- $t$-structures in (i) are called intermediate with respect to $(A, B)$. The silting subcategories in (ii) are called 2-term with respect to $S$. Clearly, if $\left(A^{\prime}, B^{\prime}\right)$ is intermediate with respect to $(A, B)$, then $(A, B)$ is intermediate with respect to $\left(\Sigma^{-1} A^{\prime}, \Sigma^{-1} B^{\prime}\right)$. The next result is a corollary of Theorems 2.2 and 2.3:

Corollary 2.4. Let $\mathrm{S}$ and $\mathrm{S}^{\prime}$ be two silting subcategories of $\mathrm{T}$. If $\mathrm{S}^{\prime}$ is 2-term with respect to $\mathrm{S}$, then $\mathrm{S}$ is 2-term with respect to $\Sigma^{-1} \mathrm{~S}^{\prime}$.

\section{Two-term silting subcategories and support $\tau$-tilting pairs}

In this section, $\mathrm{T}$ is an essentially small, idempotent complete triangulated category, and $\mathrm{S} \subseteq \mathrm{T}$ is a silting subcategory.

Remark 3.1. (i) There is a functor

$$
F: \mathrm{T} \rightarrow \operatorname{Mod} \mathrm{S},\left.\quad t \mapsto \mathrm{T}(-, t)\right|_{\mathrm{S}}
$$

sometimes known as the restricted Yoneda functor.

(ii) By Yoneda's lemma, for $M \in \operatorname{Mod} \mathrm{S}$ and $s \in \mathrm{S}$, there is a natural isomorphism

$$
\operatorname{Hom}_{\operatorname{ModS}}(\mathrm{S}(-, s), M) \stackrel{\sim}{\longrightarrow} M(s) ;
$$

see [Auslander 1974, p. 185].

(iii) By [Iyama and Yoshino 2008, Proposition 6.2(3)], the functor $F$ from (i) induces an equivalence

$$
(\mathrm{S} * \Sigma \mathrm{S}) /[\Sigma \mathrm{S}] \stackrel{\sim}{\longrightarrow} \bmod \mathrm{S} .
$$

This follows from that proposition by setting $\mathscr{X}=\mathrm{S}, \mathscr{Y}=\Sigma \mathrm{S}$, and observing that the proof works in the generality of the present paper.

Lemma 3.2. Let $\mathrm{U}$ be a full subcategory of $\mathrm{S} * \Sigma \mathrm{S}$. For $u \in \mathrm{U}$ let

$$
s_{1}^{u} \stackrel{\sigma}{\longrightarrow} s_{0}^{u} \longrightarrow u \longrightarrow \Sigma s_{1}^{u}
$$

be a distinguished triangle in $\mathrm{T}$ with $s_{0}^{u}, s_{1}^{u} \in \mathrm{S}$. Applying the functor $F$ gives a projective presentation

$$
P_{1}^{U} \stackrel{\pi^{u}}{\longrightarrow} P_{0}^{U} \longrightarrow U \longrightarrow 0
$$

in $\bmod \mathrm{S}$, and

$\mathrm{U}$ is a presilting subcategory $\Longleftrightarrow$ the class $\left\{\pi^{u} \mid u \in \mathrm{U}\right\}$ has property $(S)$. 
Proof. Clearly, $F$ applied to the distinguished triangle (2) gives the projective presentation (3).

To get the bi-implication in the last line of the lemma, first note that for $u, u^{\prime} \in \mathrm{U}$ we have

$$
\mathrm{T}\left(u, \Sigma^{\geq 2} u^{\prime}\right)=0
$$

since $u, u^{\prime} \in \mathrm{S} * \Sigma \mathrm{S}$.

By Remark 3.1(ii), the map $\operatorname{Hom}_{\bmod S}\left(\pi, F\left(u^{\prime}\right)\right)$ is the same as

$$
\mathrm{T}\left(s_{0}^{u}, u^{\prime}\right) \rightarrow \mathrm{T}\left(s_{1}^{u}, u^{\prime}\right) .
$$

So the class $\left\{\pi^{u} \mid u \in \mathrm{U}\right\}$ has property (S) if and only if the morphism (5) is surjective for all $u, u^{\prime} \in \mathrm{U}$. However, the distinguished triangle (2) induces an exact sequence

$$
\mathrm{T}\left(s_{0}^{u}, u^{\prime}\right) \longrightarrow \mathrm{T}\left(s_{1}^{u}, u^{\prime}\right) \longrightarrow \mathrm{T}\left(\Sigma^{-1} u, u^{\prime}\right) \longrightarrow \mathrm{T}\left(\Sigma^{-1} s_{0}^{u}, u^{\prime}\right),
$$

where the last module is 0 since $u^{\prime} \in \mathrm{S} * \Sigma \mathrm{S}$. So (5) is surjective if and only if $\mathrm{T}\left(\Sigma^{-1} u, u^{\prime}\right) \cong \mathrm{T}\left(u, \Sigma u^{\prime}\right)=0$. This happens for all $u, u^{\prime} \in \mathrm{U}$ if and only if $\mathrm{U}$ is presilting, because of (4).

Theorem 3.3. The functor $F: T \rightarrow$ Mod S induces a surjection

$$
\Phi: \mathrm{U} \mapsto\left(F(\mathrm{U}), \mathrm{S} \cap \Sigma^{-1} \mathrm{U}\right)
$$

from the first of the following sets to the second:

(i) Presilting subcategories of $\mathrm{T}$ which are contained in $\mathrm{S} * \Sigma \mathrm{S}$.

(ii) $\tau$-rigid pairs of $\bmod \mathrm{S}$.

It restricts to a surjection $\Psi$ from the first of the following sets to the second:

(iii) Silting subcategories of $\mathrm{T}$ which are contained in $\mathrm{S} * \Sigma \mathrm{S}$.

(iv) Support $\tau$-tilting pairs of mod S.

Proof. We need to prove

(a) The map $\Phi$ has values in $\tau$-rigid pairs of modS.

(b) The map $\Phi$ is surjective.

(c) The map $\Psi$ has values in support $\tau$-tilting pairs of $\bmod S$.

(d) The map $\Psi$ is surjective.

(a) Let $\mathrm{U}$ be a presilting subcategory of $\mathrm{T}$ which is contained in $\mathrm{S} * \Sigma \mathrm{S}$. For each $u \in \mathrm{U}$, there is a distinguished triangle $s_{1} \rightarrow s_{0} \rightarrow u \rightarrow \Sigma s_{1}$ with $s_{0}, s_{1} \in \mathrm{S}$. Lemma 3.2 says that $F$ sends the set of these triangles to a set of projective presentations (3) which has property (S), because $U$ is presilting. It remains to show that for $u \in \mathrm{U}$ and $u^{\prime} \in \mathrm{S} \cap \Sigma^{-1} \mathrm{U}$ we have $F(u)\left(u^{\prime}\right)=0$. This is again true because $F(u)\left(u^{\prime}\right)=\mathrm{T}\left(u^{\prime}, u\right)$ and $\mathrm{U}$ is presilting. 
(b) Let $(\mathrm{M}, \mathrm{E})$ be a $\tau$-rigid pair of $\bmod \mathrm{S}$. For each $m \in \mathrm{M}$ take a projective presentation

$$
P_{1} \stackrel{\pi^{m}}{\longrightarrow} P_{0} \longrightarrow m \longrightarrow 0
$$

such that the class $\left\{\pi^{m} \mid m \in \mathrm{M}\right\}$ has property (S). By Remark 3.1(ii) there is a unique morphism $f_{m}: s_{1} \rightarrow s_{0}$ in S such that $F\left(f_{m}\right)=\pi^{m}$. Moreover, $F\left(\operatorname{cone}\left(f_{m}\right)\right) \cong m$. Since (6) has property (S), it follows from Lemma 3.2 that the category

$$
\mathrm{U}_{1}:=\left\{\operatorname{cone}\left(f_{m}\right) \mid m \in \mathrm{M}\right\}
$$

is a presilting subcategory, and the inclusion $\mathrm{U}_{1} \subseteq \mathrm{S} * \Sigma \mathrm{S}$ is clear. Let $\mathrm{U}$ be the additive hull of $\mathrm{U}_{1}$ and $\Sigma \mathrm{E}$ in $\mathrm{S} * \Sigma \mathrm{S}$. Now we show that $\mathrm{U}$ is a presilting subcategory of T. Let $e \in \mathrm{E}$. Clearly we have $\mathrm{T}\left(\operatorname{cone}\left(f_{m}\right) \oplus \Sigma e, \Sigma^{2} e\right)=0$. Applying $\mathrm{T}(e,-)$ to a triangle $s_{1} \stackrel{f_{m}}{\longrightarrow} s_{0} \rightarrow \operatorname{cone}\left(f_{m}\right) \rightarrow \Sigma s_{1}$, we have an exact sequence

$$
\mathrm{T}\left(e, s_{1}\right) \stackrel{f_{m}}{\longrightarrow} \mathrm{T}\left(e, s_{0}\right) \longrightarrow \mathrm{T}\left(e, \operatorname{cone}\left(f_{m}\right)\right) \longrightarrow 0,
$$

which is isomorphic to $P_{1}(e) \stackrel{\pi^{m}}{\longrightarrow} P_{0}(e) \rightarrow m(e) \rightarrow 0$ by Remark 3.1(ii). The condition $\mathrm{M}(\mathrm{E})=0$ implies that $\mathrm{T}\left(e\right.$, cone $\left.\left(f_{m}\right)\right)=0$. Thus the assertion follows. It is clear that $\Phi(\mathrm{U})=(\mathrm{M}, \mathrm{E})$.

(c) Let $\mathrm{U}$ be a silting subcategory of $\mathrm{T}$ which is contained in $\mathrm{S} * \Sigma \mathrm{S}$.

Let $s \in \mathrm{S}$ be an object of $\operatorname{Ker} F(\mathrm{U})$, i.e., $\mathrm{T}(s, u)=0$ for each $u \in \mathrm{U}$. This implies that $\mathrm{U} \oplus \operatorname{add}(\Sigma s)$ is also a silting subcategory of $\mathrm{T}$ in $\mathrm{S} * \Sigma \mathrm{S}$. It follows from [Aihara and Iyama 2012, Theorem 2.18] that $\Sigma s$ belongs to U, whence $s$ belongs to $\Sigma^{-1} \mathrm{U}$ and hence to $\mathrm{S} \cap \Sigma^{-1} \mathrm{U}$. This shows the inclusion $\operatorname{Ker} F(\mathrm{U}) \subseteq \mathrm{S} \cap \Sigma^{-1} \mathrm{U}$. The reverse inclusion was shown in (a), so $\operatorname{Ker} F(\mathrm{U})=\mathrm{S} \cap \Sigma^{-1} \mathrm{U}$.

By Corollary 2.4, we have $\mathrm{S} \subseteq\left(\Sigma^{-1} \mathrm{U}\right) * \mathrm{U}$. In particular, for $s \in \mathrm{S}$, there is a distinguished triangle

$$
s \longrightarrow u^{0} \longrightarrow u^{1} \longrightarrow \Sigma s .
$$

Applying $F$, we obtain an exact sequence

$$
F(s) \stackrel{f}{\longrightarrow} F\left(u^{0}\right) \longrightarrow F\left(u^{1}\right) \longrightarrow 0 .
$$

For each $u \in \mathrm{U}$, we have the commutative diagram

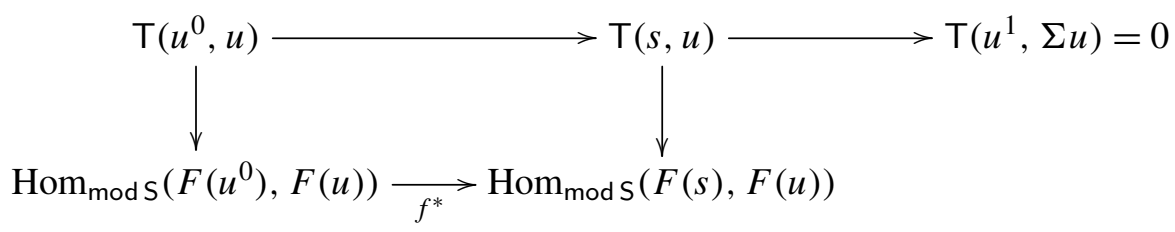


The right vertical map is induced from the Yoneda embedding, so it is bijective. It follows that $f^{*}$ is surjective, that is, $f$ is a left $F(\mathrm{U})$-approximation. Altogether, we have shown that $\Phi(\mathrm{U})$ is a support $\tau$-tilting pair of mod $\mathrm{S}$.

(d) Let $(M, E)$ be a support $\tau$-tilting pair of $\bmod S$, and let $U$ be the preimage of $(\mathrm{M}, \mathrm{E})$ under the map $\Phi$ constructed in (b).

By definition, for each $s \in \mathrm{S}$ there is an exact sequence $F(s) \stackrel{f}{\longrightarrow} F\left(u_{s}^{0}\right) \rightarrow$ $F\left(u_{s}^{1}\right) \rightarrow 0$ such that $u_{s}^{0}, u_{s}^{1} \in \mathrm{U}$ and $f$ is a left $F(\mathrm{U})$-approximation. By Yoneda's lemma, there is a unique morphism $\alpha: s \rightarrow u_{s}^{0}$ such that $F(\alpha)=f$. Form the distinguished triangle

$$
s \stackrel{\alpha}{\longrightarrow} u_{s}^{0} \longrightarrow t_{s} \longrightarrow \Sigma s .
$$

Let $\widetilde{U}$ be the additive closure of $U$ and $\left\{t_{s} \mid s \in U\right\}$. We claim that $\widetilde{U}$ is a silting subcategory of $\mathrm{T}$ contained in $\mathrm{S} * \Sigma \mathrm{S}$ such that $\Phi(\widetilde{\mathrm{U}})=(\mathrm{M}, \mathrm{E})$.

First, $t_{s} \in u_{s}^{0} * \Sigma s \subseteq \mathrm{S} * \Sigma \mathrm{S}$. Therefore, $\widetilde{\mathrm{U}} \subseteq \mathrm{S} * \Sigma \mathrm{S}$.

Second, by applying $F$ to the triangle (9), we see that $F\left(t_{s}\right)$ and $F\left(u_{s}^{1}\right)$ are isomorphic in mod $\mathrm{S}$. For $u \in \mathrm{U}$, consider the following commutative diagram.

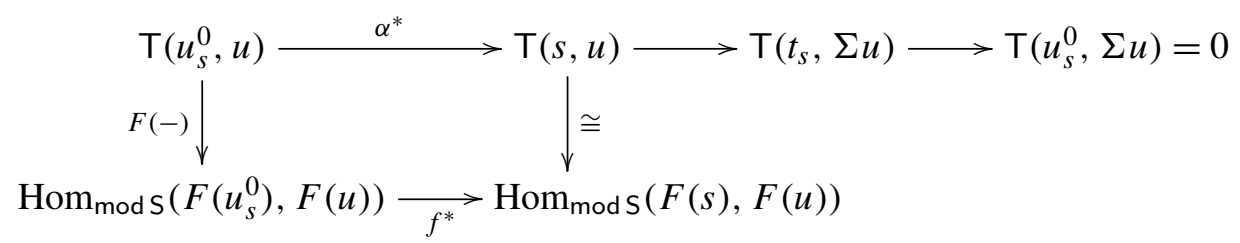

By Remark 3.1(iii), the map $F(-)$ is surjective. Because $f$ is a left $F(\mathrm{U})$-approximation, $f^{*}$ is also surjective. So $\alpha^{*}$ is surjective too, implying that $\mathrm{T}\left(t_{s}, \Sigma u\right)=0$. On the other hand, applying $\mathrm{T}(u,-)$ to the triangle (9), we obtain an exact sequence

$$
\mathrm{T}\left(u, \Sigma u_{s}^{0}\right) \longrightarrow \mathrm{T}\left(u, \Sigma t_{s}\right) \longrightarrow \mathrm{T}\left(u, \Sigma^{2} s\right) .
$$

The two outer terms are trivial, hence so is the middle term. Moreover, if $s^{\prime} \in \mathrm{S}$, then applying $\mathrm{T}\left(t_{s^{\prime}},-\right)$ to the triangle (9) gives an exact sequence

$$
\mathrm{T}\left(t_{s^{\prime}}, \Sigma u_{s}^{0}\right) \longrightarrow \mathrm{T}\left(t_{s^{\prime}}, \Sigma t_{s}\right) \longrightarrow \mathrm{T}\left(t_{s^{\prime}}, \Sigma^{2} s\right) .
$$

The two outer terms are trivial, hence so is the middle term. It follows that $\widetilde{U}$ is presilting. It is then silting because it generates $\mathrm{S}$.

Thirdly, $F(\widetilde{\mathrm{U}})=F(\mathrm{U})$ because $F\left(t_{s}\right) \cong F\left(u_{s}^{1}\right)$.

Finally, $S \cap \Sigma^{-1} \widetilde{U}=E$. This is because $S \cap \Sigma^{-1} \widetilde{U} \supseteq S \cap \Sigma^{-1} U=E$ and $\mathrm{S} \cap \Sigma^{-1} \widetilde{\mathrm{U}} \subseteq \operatorname{Ker} F(\mathrm{U})=\mathrm{E}$.

Theorem 3.4. Assume that each object of $\mathrm{S} * \Sigma \mathrm{S}$ can be written as the direct sum of indecomposable objects which are unique up to isomorphism. Then the maps $\Phi$ and $\Psi$ defined in Theorem 3.3 are bijective. 
Proof. It suffices to show the injectivity of $\Phi$.

By Remark 3.1(iii), when we apply the functor $F: S * \Sigma S \rightarrow \bmod S$, we are in effect forgetting the indecomposable direct summands which are in $\Sigma$ S. So if $F(u) \cong F\left(u^{\prime}\right)$ for $u, u^{\prime} \in \mathrm{S} * \Sigma \mathrm{S}$, then there is an isomorphism $u \oplus \Sigma s \cong u^{\prime} \oplus \Sigma s^{\prime}$ for some $s, s^{\prime} \in \mathrm{S}$. By the assumption in the theorem, if we assume that $u$ and $u^{\prime}$ do not have direct summands in $\Sigma \mathrm{S}$, then $u \cong u^{\prime}$.

Now let $\mathrm{U}$ and $\mathrm{U}^{\prime}$ be two presilting subcategories of $\mathrm{T}$ contained in $\mathrm{S} * \Sigma \mathrm{S}$ such that $\Phi(\mathrm{U})=\Phi\left(\mathrm{U}^{\prime}\right)$. Let $\mathrm{U}_{1}$ and $\mathrm{U}_{1}^{\prime}$ be respectively the full subcategories of $\mathrm{U}$ and $\mathrm{U}^{\prime}$ consisting of objects without direct summands in $\Sigma \mathrm{S}$. Then $\mathrm{U}=\mathrm{U}_{1} \oplus(\mathrm{U} \cap \Sigma \mathrm{S})$ and $\mathrm{U}^{\prime}=\mathrm{U}_{1}^{\prime} \oplus\left(\mathrm{U}^{\prime} \cap \Sigma S\right)$. Since $\Phi(\mathrm{U})=\Phi\left(\mathrm{U}^{\prime}\right)$, it follows that $F\left(\mathrm{U}_{1}\right)=F\left(\mathrm{U}_{1}^{\prime}\right)$ and $\mathrm{U} \cap \Sigma \mathrm{S}=\mathrm{U}^{\prime} \cap \Sigma \mathrm{S}$. The first equality, by the above argument, implies that $\mathrm{U}_{1}=\mathrm{U}_{1}^{\prime}$. Therefore $U=U^{\prime}$, which shows the injectivity of $\Phi$.

\section{The Hom-finite Krull-Schmidt silting object case}

In this section, $\mathbb{k}$ is a commutative ring, $\mathrm{T}$ is a triangulated category which is essentially small, Krull-Schmidt, $\mathbb{k}$-linear and Hom-finite, and $s \in \mathrm{T}$ is a basic silting object.

We write $E=\mathrm{T}(s, s)$ for the endomorphism ring and $\mathrm{S}=\operatorname{add}(s)$ for the associated silting subcategory.

Remark 4.1. (i) We write $\operatorname{Mod} E$ for the abelian category of right $E$-modules, $\bmod E$ for the full subcategory of finitely presented modules, and prj $E$ for the full subcategory of finitely generated projective modules.

(ii) Since $s$ is an additive generator of $\mathrm{S}$, there is an equivalence

$$
G: \operatorname{Mod} \mathrm{S} \stackrel{\sim}{\longrightarrow} \operatorname{Mod} E, \quad M \mapsto M(s),
$$

which restricts to an equivalence

$$
\bmod \mathrm{S} \stackrel{\sim}{\longrightarrow} \bmod E, \quad M \mapsto M(s) .
$$

This permits us to move freely between the " $E$-picture" and the "S-picture" which was used in the previous section.

(iii) The restricted Yoneda functor $F$ from the S-picture corresponds to the functor

$$
\mathrm{T} \rightarrow \operatorname{Mod} E, \quad t \mapsto \mathrm{T}(s, t)
$$

in the $E$-picture.

(iv) By [Auslander 1974, Proposition 2.2(e)] the functor $t \mapsto \mathrm{T}(s, t)$ from (iii) restricts to an equivalence

$$
Y: \mathrm{S} \stackrel{\sim}{\longrightarrow} \operatorname{prj} E .
$$


Since $S=\operatorname{add}(s)$ is closed under direct sums and summands, it is KrullSchmidt, and it follows that so is prj $E$.

(v) By Remark 3.1(iii) the functor $t \mapsto \mathrm{T}(s, t)$ from (iii) induces an equivalence

$$
(\mathrm{S} * \Sigma \mathrm{S}) /[\Sigma \mathrm{S}] \stackrel{\sim}{\longrightarrow} \bmod E .
$$

Since $\mathrm{S} * \Sigma \mathrm{S}$ is obviously closed under direct sums, and under direct summands by Lemma 2.1 , it is Krull-Schmidt. Hence so is $(\mathrm{S} * \Sigma \mathrm{S}) /[\Sigma \mathrm{S}]$ and it follows that so is $\bmod E$.

(vi) The additive category prj $E$ is Krull-Schmidt by part (iv) and has additive generator $E_{E}$. The same is hence true for ( $\operatorname{prj} E$ )/[add $e E$ ] for each idempotent $e \in E$. It is not hard to check that the endomorphism ring of $E_{E}$ in $(\operatorname{prj} E) /[\operatorname{add} e E]$ is $E / E e E$, so there is an equivalence of categories

$$
(\operatorname{prj} E) /[\operatorname{add} e E] \stackrel{\sim}{\rightarrow} \operatorname{prj}(E / E e E) .
$$

In particular, $\operatorname{prj}(E / E e E)$ is Krull-Schmidt.

The following result is essentially already in [Aihara 2013, Proposition 2.16], [Fei and Derksen 2011, start of Section 5], and [Wei 2013, Proposition 6.1], all of which give triangulated versions of Bongartz's classic proof:

Lemma 4.2 (Bongartz completion). Let $u \in \mathrm{S} * \Sigma \mathrm{S}$ be a presilting object. Then there exists an object $u^{\prime} \in \mathrm{S} * \Sigma \mathrm{S}$ such that $u \oplus u^{\prime}$ is a silting object.

Proof. This has essentially the same proof as classic Bongartz completion: Since T is Hom-finite over the commutative ring $\mathbb{k}$, there is a right $\operatorname{add}(u)$-approximation $u_{0} \rightarrow \Sigma s$. This gives a distinguished triangle $s \rightarrow u^{\prime} \rightarrow u_{0} \rightarrow \Sigma s$, and it is straightforward to check that $u^{\prime}$ has the desired properties.

The following result is essentially already contained in [Fei and Derksen 2011, Theorem 5.4]:

Proposition 4.3. Let $u \in \mathrm{S} * \Sigma \mathrm{S}$ be a basic presilting object. Then

$$
u \text { is a silting object } \Longleftrightarrow \#_{\mathrm{T}}(u)=\#_{\mathrm{T}}(s) \text {. }
$$

Proof. The implication $\Longrightarrow$ is immediate from [Aihara and Iyama 2012, Theorem 2.27], and $\Longleftarrow$ is a straightforward consequence of that theorem and Lemma 4.2.

As a consequence, we have:

Corollary 4.4. Let $\mathrm{U}$ be a presilting subcategory of $\mathrm{T}$ contained in $\mathrm{S} * \Sigma \mathrm{S}$. Then there exists $u \in \mathrm{U}$ such that $\mathrm{U}=\operatorname{add}(u)$. 
Proof. Suppose on the contrary that $\mathrm{U} \neq \operatorname{add}(u)$ for each $u \in \mathrm{U}$. Then $\mathrm{U}$ contains infinitely many isomorphism classes of indecomposable objects. In particular, there is a basic presilting object $u \in \mathrm{U}$ such that $\#_{\mathrm{T}}(u)=\#_{\mathrm{T}}(s)+1$. By Lemma 4.2, there is an object $u^{\prime} \in \mathrm{T}$ such that $u \oplus u^{\prime}$ is a basic silting object of T. Therefore, $\#_{\mathrm{T}}(s)+1=\#_{\mathrm{T}}(u) \leq \#_{\mathrm{T}}\left(u \oplus u^{\prime}\right)=\#_{\mathrm{T}}(s)$, a contradiction. Here the last equality follows from Proposition 4.3.

Theorem 3.3 in the current setting combined with Corollary 4.4 immediately yields the following result. For an object $u$ of $\mathrm{S} * \Sigma \mathrm{S}$, let $\Sigma u_{1}$ be its maximal direct summand in $\Sigma$ S.

Theorem 4.5. The assignment

$$
u \mapsto\left(\operatorname{add}(F(u)), \operatorname{add}\left(u_{1}\right)\right)
$$

defines a bijection from the first of the following sets to the second:

(i) Basic presilting objects of $\mathrm{T}$ which are in $\mathrm{S} * \Sigma \mathrm{S}$, modulo isomorphism.

(ii) $\tau$-rigid pairs of $\bmod \mathrm{S}$.

It restricts to a bijection from the first of the following sets to the second:

(iii) Basic silting objects of $\mathrm{T}$ which are in $\mathrm{S} * \Sigma \mathrm{S}$, modulo isomorphism.

(iv) Support $\tau$-tilting pairs of mod S.

As a consequence, if $(\mathrm{M}, \mathrm{E})$ is a $\tau$-rigid pair of $\bmod \mathrm{S}$, then there is an S-module $M$ such that $\mathrm{M}=\operatorname{add}(M)$.

Next we move to the $E$-picture. Recall from Remark 4.1(ii) and (iv) that there are equivalences $G: \operatorname{Mod} S \stackrel{\sim}{\longrightarrow} \operatorname{Mod} E$ and $Y: S \stackrel{\sim}{\longrightarrow} \operatorname{prj} E$.

Theorem 4.6. An E-module $U$ is a support $\tau$-tilting module if and only if the pair

$$
\left(G^{-1}(\operatorname{add}(U)), Y^{-1}(\operatorname{add}(e E))\right)
$$

is a support $\tau$-tilting pair of mod $\mathrm{S}$ for some idempotent $e \in E$.

Consequently, the functor $\mathrm{T}(s,-): \mathrm{T} \rightarrow \operatorname{Mod} E$ induces a bijection from the first of the following sets to the second:

(i) Basic silting objects of $\mathrm{T}$ which are in $\mathrm{S} * \Sigma \mathrm{S}$, modulo isomorphism.

(ii) Basic support $\tau$-tilting modules of $\bmod E$, modulo isomorphism.

Proof. We only prove the first assertion. The proof is divided into three parts. Let $u_{p} \in \mathrm{S} * \Sigma \mathrm{S}$ be such that $u_{p}$ has no direct summand in $\Sigma \mathrm{S}$ and $F\left(u_{p}\right)=G^{-1}(U)$.

(a) It is clear that $U$ is a $\tau$-rigid $E$-module if and only if $G^{-1}(\operatorname{add}(U))$ is a $\tau$-rigid subcategory of modS. 
(b) Let $e$ be an idempotent of $E$ and let $u_{1} \in \mathrm{S}$ be such that $Y\left(u_{1}\right)=e E$. We have

$$
\begin{aligned}
U e & \cong \operatorname{Hom}_{E}(e E, U) \\
& =\operatorname{Hom}_{\operatorname{Mod} S}\left(\mathrm{~S}\left(-, u_{1}\right), F\left(u_{p}\right)\right) \\
& \cong F\left(u_{p}\right)\left(u_{1}\right) \quad \text { Remark 3.1(ii). }
\end{aligned}
$$

Therefore $U e=0$ if and only if $M\left(u^{\prime}\right)=0$ for each $M \in \operatorname{add}\left(F\left(u_{p}\right)\right)=G^{-1}(\operatorname{add}(U))$ and each $u^{\prime} \in \operatorname{add}\left(u_{1}\right)=Y^{-1}(\operatorname{add}(e E))$.

(c) Suppose that $\left(G^{-1}(\operatorname{add}(U)), Y^{-1}(\operatorname{add}(e E))\right)$ is a $\tau$-rigid pair. Let $u$ be the corresponding basic presilting object of $\mathrm{T}$ as in Theorem 4.5. More precisely, let $u=u_{p} \oplus \Sigma u_{1}$, where $u_{p}$ and $u_{1}$ are as above. Then

$\left(G^{-1}(\operatorname{add}(U)), Y^{-1}(\operatorname{add}(e E))\right)$ is a support $\tau$-tilting pair

$$
\begin{array}{lr}
\Longleftrightarrow u \text { is a silting object } & \text { Theorem 4.5 } \\
\Longleftrightarrow \#_{\mathrm{T}}(u)=\#_{\mathrm{T}}(s) & \text { Proposition 4.3 } \\
\Longleftrightarrow \#_{\mathrm{S} * \Sigma \mathrm{S}}(u)=\#_{\mathrm{S}}(s) & \\
\Longleftrightarrow \#_{\mathrm{S} * \Sigma \mathrm{S}}(u)=\#_{\operatorname{prj} E}(E) & \text { Remark 4.1(iv) } \\
\Longleftrightarrow \#_{(\mathrm{S} * \Sigma \mathrm{S}) /[\Sigma \mathrm{S}]}(u)+\#_{\mathrm{S} * \Sigma \mathrm{S}}\left(\Sigma u_{1}\right)=\#_{\mathrm{prj} E}(E) & \\
\Longleftrightarrow \#_{\bmod E}(U)+\#_{\operatorname{prj} E}(e E)=\#_{\mathrm{prj} E}(E) & \text { Remark 4.1(iv), (v) } \\
\Longleftrightarrow \#_{\bmod E}(U)=\#_{\operatorname{prj} E}(E)-\#_{\operatorname{prj} E}(e E) & \\
\Longleftrightarrow \#_{\bmod E}(U)=\#_{(\operatorname{prj} E) /[\operatorname{add} e E]}(E) & \\
\Longleftrightarrow \#_{\bmod E}(U)=\#_{\operatorname{prj}(E / E e E)}(E / E e E) & \text { Remark 4.1(vi) } \\
\Longleftrightarrow U \text { is a support } \tau \text {-tilting module. }
\end{array}
$$

\section{Support $\tau$-tilting pairs and torsion classes}

In this section $\mathbb{k}$ is a commutative noetherian local ring and $\mathrm{C}$ is an essentially small, Krull-Schmidt, $\mathbb{k}$-linear and Hom-finite category.

The main result in this section is the following:

Theorem 5.1. There is a bijection $\mathrm{M} \mapsto \mathrm{Fac} \mathrm{M}$ from the first of the following sets to the second:

(i) Support $\tau$-tilting pairs (M, E) of $\bmod C$.

(ii) Finitely generated torsion classes $\mathrm{T}$ of Mod $\mathrm{C}$ such that each finitely generated projective $\mathrm{C}$-module has a left $\mathrm{P}(\mathrm{T})$-approximation.

We start with the following observation: 
Lemma 5.2. Let $\mathrm{M}$ be a subcategory of $\bmod \mathrm{C}$. The following conditions are equivalent:

(i) $\mathrm{M}$ is $\tau$-rigid.

(ii) $\operatorname{Ext}_{\text {Mod C }}^{1}(\mathrm{M}, \operatorname{Fac} M)=0$.

(iii) Each $m \in \mathrm{M}$ has a minimal projective presentation

$$
0 \longrightarrow \Omega^{2} m \stackrel{d_{2}}{\longrightarrow} P_{1} \stackrel{d_{1}}{\longrightarrow} P_{0} \longrightarrow m \longrightarrow 0
$$

such that for each $m^{\prime} \in \mathrm{M}$ and each morphism $f: P_{1} \rightarrow m^{\prime}$, there exist morphisms $a: P_{0} \rightarrow m^{\prime}$ and $b: P_{1} \rightarrow \Omega^{2} m$ such that $f=a d_{1}+f d_{2} b$.

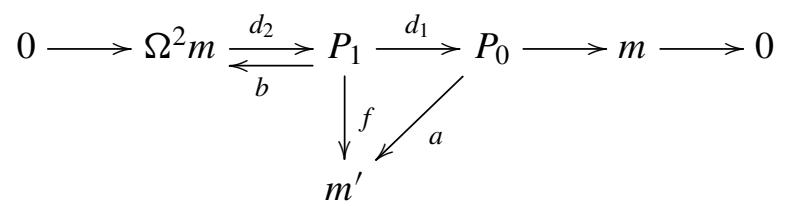

Proof. (i) $\Longrightarrow$ (ii): For each $m \in \mathrm{M}$, there exists a projective presentation $P_{1} \stackrel{\pi}{\longrightarrow} P_{0} \rightarrow$ $m \rightarrow 0$ such that $\operatorname{Hom}_{\operatorname{Mod} C}\left(\pi, m^{\prime}\right)$ is surjective for each $m^{\prime} \in \mathrm{M}$. Let $n \in$ Fac M be given and pick an epimorphism $p: m^{\prime} \rightarrow n$ with $m^{\prime} \in \mathrm{M}$. To show $\operatorname{Ext}_{\text {Mod C }}^{1}(m, n)=0$, it is enough to show that each $f \in \operatorname{Hom}_{\operatorname{Mod} C}\left(P_{1}, n\right)$ factors through $\pi$. Since $p$ is an epimorphism and $P_{1}$ is projective, there exists $g: P_{1} \rightarrow m^{\prime}$ such that $f=p g$. Then there exists $h: P_{0} \rightarrow m^{\prime}$ such that $g=h \pi$, by the property of $\pi$.

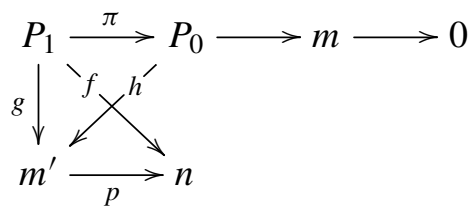

Thus $f=p h \pi$, and we have the assertion.

(ii) $\Longrightarrow$ (iii): For each $m \in \mathrm{M}$, take a minimal projective presentation $0 \rightarrow \Omega^{2} m \stackrel{d_{2}}{\longrightarrow}$ $P_{1} \stackrel{d_{1}}{\longrightarrow} P_{0} \rightarrow m \rightarrow 0$. Let $m^{\prime} \in \mathrm{M}$ and $f: P_{1} \rightarrow m^{\prime}$ be given, set $n:=\operatorname{Im}\left(f d_{2}\right)$ and let $0 \rightarrow n \stackrel{\iota}{\rightarrow} m^{\prime} \stackrel{\pi}{\longrightarrow} n^{\prime} \rightarrow 0$ be an exact sequence. Then $\pi f: P_{1} \rightarrow n^{\prime}$ factors through $P_{1} \rightarrow \operatorname{Im} d_{1}$. Since $n^{\prime} \in \operatorname{Fac} \mathrm{M}$ and $\operatorname{Ext}_{\operatorname{Mod} C}^{1}(m, \operatorname{Fac} \mathrm{M})=0$, there exists $g: P_{0} \rightarrow n^{\prime}$ such that $g d_{1}=\pi f$.

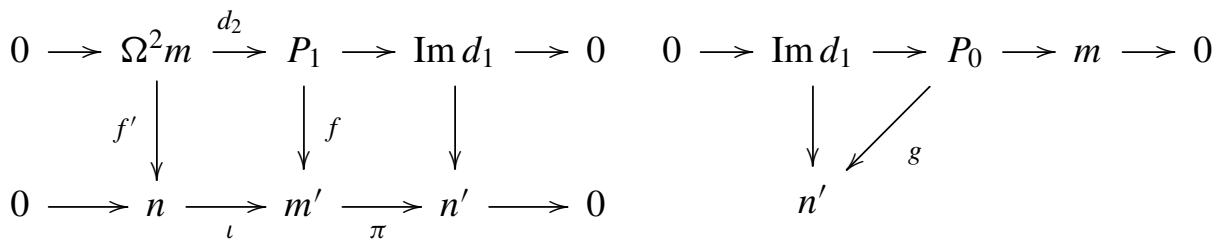


Since $\pi$ is an epimorphism and $P_{0}$ is projective, there exists $a: P_{0} \rightarrow m^{\prime}$ such that $g=\pi a$. Since $\pi\left(f-a d_{1}\right)=0$, there exists $h: P_{1} \rightarrow n$ such that $f=a d_{1}+\iota h$. Since $f^{\prime}$ is surjective (by definition of $n$ ) and $P_{1}$ is projective, there exists $b: P_{1} \rightarrow \Omega^{2} m$ such that $h=f^{\prime} b$.

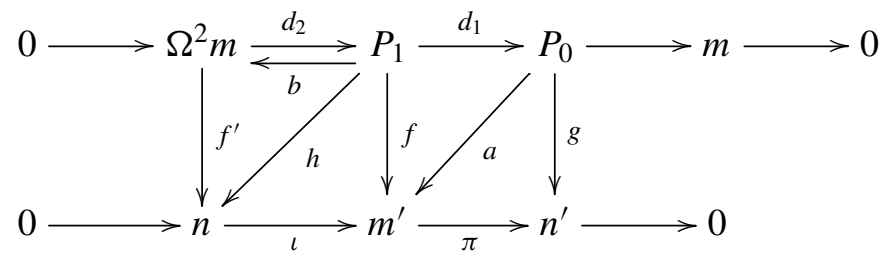

Then we have $f=a d_{1}+\iota f^{\prime} b=a d_{1}+f d_{2} b$.

(iii) $\Longrightarrow$ (i): For each $m \in M$, take a minimal projective presentation $0 \rightarrow \Omega^{2} m \stackrel{d_{2}}{\longrightarrow}$ $P_{1} \stackrel{d_{1}}{\rightarrow} P_{0} \rightarrow m \rightarrow 0$ satisfying the assumption in (iii). We need to show that each $f: P_{1} \rightarrow m^{\prime}$ with $m^{\prime} \in \mathrm{M}$ factors through $d_{1}$. By our assumption, there exist $a: P_{0} \rightarrow m^{\prime}$ and $b: P_{1} \rightarrow \Omega^{2} m$ such that $f=a d_{1}+f d_{2} b$. Applying our assumption to $f d_{2} b: P_{1} \rightarrow m^{\prime}$, there exist $a^{\prime}: P_{0} \rightarrow m^{\prime}$ and $b^{\prime}: P_{1} \rightarrow \Omega^{2} m$ such that $f d_{2} b=a^{\prime} d_{1}+f d_{2} b d_{2} b^{\prime}$. Thus $f=\left(a+a^{\prime}\right) d_{1}+f d_{2} b d_{2} b^{\prime}$. Repeating a similar argument gives

$\operatorname{Hom}_{M o d C}\left(P_{1}, m\right)=\operatorname{Hom}_{\operatorname{Mod} C}\left(P_{0}, m\right) d_{1}+\operatorname{Hom}_{\operatorname{Mod} C}\left(P_{1}, m\right)\left(\operatorname{rad}_{\operatorname{End}} \operatorname{Mod} \mathrm{C}\left(P_{1}\right)\right)^{n}$

for each $n \geq 1$, since $d_{2} \in \operatorname{rad} \operatorname{Hom}_{\operatorname{Mod} \mathrm{C}}\left(\Omega^{2} m, P_{1}\right)$. Since C is Hom-finite over $\mathbb{k}$, we have $\left(\operatorname{rad} \operatorname{End}_{\operatorname{Mod} C}\left(P_{1}\right)\right)^{\ell} \subset \operatorname{End}_{\operatorname{Mod} C}\left(P_{1}\right)(\operatorname{rad} \mathbb{k})$ for sufficiently large $\ell$. Thus we have

$$
\operatorname{Hom}_{M o d C}\left(P_{1}, m\right)=\bigcap_{n \geq 0}\left(\operatorname{Hom}_{M o d C}\left(P_{0}, m\right) d_{1}+\operatorname{Hom}_{\operatorname{Mod} C}\left(P_{1}, m\right)(\operatorname{rad} \mathbb{k})^{n}\right) .
$$

The right-hand side is equal to $\operatorname{Hom}_{\operatorname{Mod} C}\left(P_{0}, m\right) d_{1}$ itself by Krull's intersection theorem [Matsumura 1989].

Proposition 5.3. Let $(\mathrm{M}, \mathrm{E})$ be a support $\tau$-tilting pair of $\bmod \mathrm{C}$. Then $\mathrm{Fac} \mathrm{M}$ is a finitely generated torsion class with $\mathrm{P}(\mathrm{Fac} \mathrm{M})=\mathrm{M}$.

Proof. (i) We show that Fac M is a torsion class. Clearly Fac M is closed under factor modules. We show that Fac $\mathrm{M}$ is closed under extensions. Let $0 \rightarrow x \rightarrow y \stackrel{f}{\rightarrow} z \rightarrow 0$ be an exact sequence in Mod $C$ such that $x, z \in$ Fac M. Take an epimorphism $p: m \rightarrow z$ with $m \in \mathrm{M}$. Since $\operatorname{Ext}_{\text {Mod C }}^{1}(m, x)=0$ by Lemma 5.2(ii), we have that $p$ factors through $f$. Thus we have an epimorphism $x \oplus m \rightarrow y$, and $y \in$ Fac $\mathrm{M}$ holds. Hence Fac M is a torsion class. 
(ii) Since $\operatorname{Ext}_{\text {Mod C }}^{1}(M, F a c M)=0$ by Lemma 5.2(ii), each object in $M$ is Extprojective in Fac M. It remains to show that if $n$ is an Ext-projective object in Fac M, then $n \in \mathrm{M}$. Let $P_{1} \stackrel{f}{\longrightarrow} P_{0} \stackrel{e}{\longrightarrow} n \rightarrow 0$ be a projective presentation. Since $\mathrm{M}$ is support $\tau$-tilting, there exist exact sequences $P_{i} \stackrel{g_{i}}{\longrightarrow} m_{i} \stackrel{h_{i}}{\longrightarrow} m_{i}^{\prime} \rightarrow 0$ with $m_{i}, m_{i}^{\prime} \in \mathrm{M}$ and a left M-approximation $g_{i}$ for $i=0,1$.

Let $\overline{\mathrm{C}}:=\mathrm{C} /$ ann $\mathrm{M}$ for the annihilator ideal ann $\mathrm{M}$ of $\mathrm{M}$ and $\overline{P_{i}}:=P_{i} \otimes_{\mathrm{C}} \overline{\mathrm{C}}$. Then we have induced exact sequences $0 \rightarrow \overline{P_{i}} \stackrel{g_{i}}{\longrightarrow} m_{i} \stackrel{h_{i}}{\longrightarrow} m_{i}^{\prime} \rightarrow 0$ for $i=0,1$ and $\overline{P_{1}} \stackrel{f}{\longrightarrow} \overline{P_{0}} \stackrel{e}{\longrightarrow} n \rightarrow 0$. We have a commutative diagram

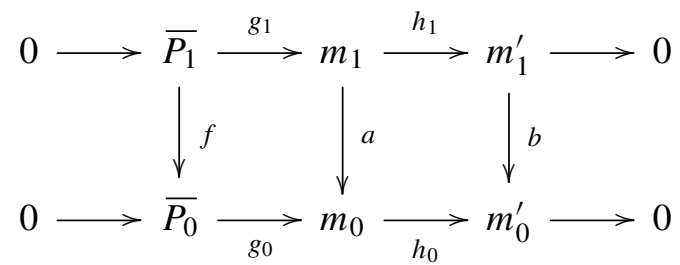

of exact sequences. Taking a mapping cone, we have an exact sequence

$$
0 \longrightarrow \overline{P_{1}} \stackrel{\left[\begin{array}{c}
g_{1} \\
f
\end{array}\right]}{\longrightarrow} m_{1} \oplus \overline{P_{0}} \stackrel{\left[\begin{array}{cc}
h_{1} & 0 \\
a & -g_{0}
\end{array}\right]}{\longrightarrow} m_{1}^{\prime} \oplus m_{0} \stackrel{\left[b-h_{0}\right]}{\longrightarrow} m_{0}^{\prime} \longrightarrow 0 .
$$

Since $\operatorname{Ext}_{\text {Mod C }}^{1}\left(m_{0}^{\prime}, n\right)=0$ by Lemma 5.2(ii), we have the following commutative diagram.

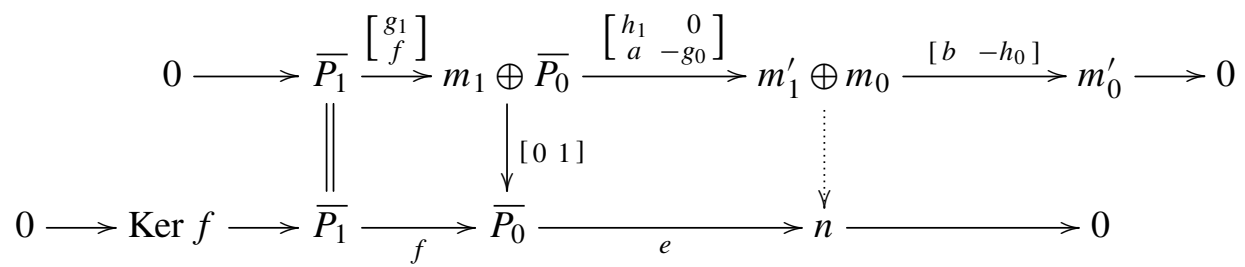

Taking a mapping cone, we have an exact sequence

$$
0 \longrightarrow \overline{P_{1}} \oplus \operatorname{Ker} f \longrightarrow m_{1} \oplus \overline{P_{0}} \oplus \overline{P_{1}} \longrightarrow m_{1}^{\prime} \oplus m_{0} \oplus \overline{P_{0}} \longrightarrow m_{0}^{\prime} \oplus n \longrightarrow 0 .
$$

Cancelling a direct summand of the form $\overline{P_{1}} \stackrel{\left[\begin{array}{l}0 \\ 1\end{array}\right]}{\longrightarrow} \overline{P_{0}} \oplus \overline{P_{1}} \stackrel{\left[\begin{array}{ll}1 & 0\end{array}\right]}{\longrightarrow} \overline{P_{0}}$, we have an exact sequence

$$
0 \longrightarrow \operatorname{Ker} f \longrightarrow m_{1} \stackrel{c}{\longrightarrow} m_{1}^{\prime} \oplus m_{0} \stackrel{d}{\longrightarrow} m_{0}^{\prime} \oplus n \longrightarrow 0 .
$$

Since $\operatorname{Im} c \in \operatorname{Fac} \mathrm{M}$ and $m_{0}^{\prime} \oplus n$ is Ext-projective in Fac M, the epimorphism $d$ splits. Thus $n \in \mathrm{M}$ as desired.

Now we are ready to prove Theorem 5.1. 
Let $\mathrm{M}$ be a support $\tau$-tilting subcategory of $\bmod \mathrm{C}$. By definition, each representable $\mathrm{C}$-module has a left $\mathrm{M}$-approximation. Since $\mathrm{P}(\mathrm{Fac} M)=\mathrm{M}$ by Proposition 5.3, the map $M \mapsto$ Fac $M$ is well-defined from the set (i) to the set (ii), and it is injective.

We show that the map is surjective. For $\mathrm{T}$ in the set described in (ii), let $\mathrm{E}:=\bigcap_{m \in \mathrm{T}} \operatorname{Ker} m$ and $\mathrm{M}:=\mathrm{P}(\mathrm{T})$. We will show that $(\mathrm{M}, \mathrm{E})$ is a support $\tau$-tilting pair of $\bmod C$. Since $\operatorname{Ext}_{\text {Mod }}^{1}(M, T)=0$ and Fac $M \subset T$, it follows from Lemma 5.2 that $\mathrm{M}$ is $\tau$-rigid. For $s \in \mathrm{C}$, take a left $\mathrm{M}$-approximation $\mathrm{C}(-, s) \stackrel{f}{\rightarrow} m$.

It remains to show Coker $f \in$ M. Since Coker $f \in \mathrm{T}$, we only have to show $\operatorname{Ext}_{\text {Mod C }}^{1}\left(\right.$ Coker $\left.f, m^{\prime}\right)=0$ for each $m^{\prime} \in \mathrm{M}$. Let $f=\imath \pi$ for $\pi: \mathrm{C}(-, s) \rightarrow \operatorname{Im} f$ and $\iota: \operatorname{Im} f \rightarrow m$. Applying $\operatorname{Hom}_{\operatorname{Mod} C}\left(-, m^{\prime}\right)$ to the exact sequence $0 \rightarrow \operatorname{Im} f \stackrel{\iota}{\rightarrow}$ $m \rightarrow$ Coker $f \rightarrow 0$, we have an exact sequence

$\operatorname{Hom}_{\operatorname{Mod} C}\left(m, m^{\prime}\right) \stackrel{\iota^{*}}{\rightarrow} \operatorname{Hom}_{\operatorname{Mod} C}\left(\operatorname{Im} f, m^{\prime}\right)$

$$
\rightarrow \operatorname{Ext}_{\text {Mod C }}^{1}\left(\operatorname{Coker} f, m^{\prime}\right) \rightarrow \operatorname{Ext}_{\text {Mod C }}^{1}\left(m, m^{\prime}\right)=0 .
$$

Let $g: \operatorname{Im} f \rightarrow m^{\prime}$ be a morphism in Mod C. Since $f$ is a left M-approximation, there exists $h: m \rightarrow m^{\prime}$ such that $g \pi=h f$. Then $g=h \iota$. Thus $\iota^{*}: \operatorname{Hom}_{\operatorname{Mod} \mathrm{C}}\left(m, m^{\prime}\right) \rightarrow$ $\operatorname{Hom}_{\text {Mod C }}\left(\operatorname{Im} f, m^{\prime}\right)$ is surjective, and we have $\operatorname{Ext}_{\text {Mod C }}^{1}\left(\right.$ Coker $\left.f, m^{\prime}\right)=0$. Consequently we have Coker $f \in \mathrm{P}(\mathrm{T})=\mathrm{M}$. Thus the assertion follows.

\section{References}

[Adachi et al. 2014] T. Adachi, O. Iyama, and I. Reiten, " $\tau$-tilting theory”, Compositio Math. 150:3 (2014), 415-452. MR 3187626 Zbl 06293659

[Aihara 2013] T. Aihara, “Tilting-connected symmetric algebras”, Algebr. Represent. Theory 16:3 (2013), 873-894. MR 3049676 Zbl 06175500

[Aihara and Iyama 2012] T. Aihara and O. Iyama, "Silting mutation in triangulated categories", J. London Math. Soc. (2) 85:3 (2012), 633-668. MR 2927802 Zbl 1271.18011

[Auslander 1974] M. Auslander, "Representation theory of Artin algebras, I", Comm. Algebra 1 (1974), 177-268. MR 50 \#2240 Zbl 0285.16028

[Bass 1968] H. Bass, Algebraic K-theory, W. A. Benjamin, New York-Amsterdam, 1968. MR 40 \#2736 Zbl 0174.30302

[Beligiannis and Reiten 2007] A. Beligiannis and I. Reiten, Homological and homotopical aspects of torsion theories, Mem. Amer. Math. Soc. 883, American Mathematical Society, Providence, 2007. MR 2009e:18026 Zbl 1124.18005

[Bondarko 2010] M. V. Bondarko, "Weight structures vs. $t$-structures; weight filtrations, spectral sequences, and complexes (for motives and in general)", J. K-Theory 6:3 (2010), 387-504. MR 2746283 Zbl 05862078

[Fei and Derksen 2011] J. Fei and H. Derksen, “General presentation of algebras", preprint, 2011. arXiv 0911.4913v2

[Happel et al. 1996] D. Happel, I. Reiten, and S. O. Smalø, Tilting in abelian categories and quasitilted algebras, Mem. Amer. Math. Soc. 575, American Mathematical Society, Providence, RI, 1996. MR 97j:16009 Zbl 0849.16011 
[Iyama and Yoshino 2008] O. Iyama and Y. Yoshino, "Mutation in triangulated categories and rigid Cohen-Macaulay modules", Invent. Math. 172:1 (2008), 117-168. MR 2008k:16028 Zbl 1140.18007

[Keller 2013] B. Keller, "The periodicity conjecture for pairs of Dynkin diagrams", Ann. of Math. (2) 177:1 (2013), 111-170. MR 2999039 Zbl 06146418

[Matsumura 1989] H. Matsumura, Commutative ring theory, 2nd ed., Cambridge Stud. in Adv. Math. 8, Cambridge University Press, Cambridge, 1989. MR 90i:13001 Zbl 0666.13002

[Mendoza Hernández et al. 2013] O. Mendoza Hernández, E. C. Sáenz Valadez, V. Santiago Vargas, and M. J. Souto Salorio, "Auslander-Buchweitz context and co-t-structures", Appl. Categ. Structures 21:5 (2013), 417-440. MR 3097052 Zbl 1291.18017

[Pauksztello 2008] D. Pauksztello, "Compact corigid objects in triangulated categories and co- $t$ structures”, Cent. Eur. J. Math. 6:1 (2008), 25-42. MR 2009d:18020 Zbl 1152.18009

[Wei 2013] J. Wei, “Semi-tilting complexes", Israel J. Math. 194:2 (2013), 871-893. MR 3047094 Zbl 1286.16011

[Woolf 2010] J. Woolf, "Stability conditions, torsion theories and tilting”, J. London Math. Soc. (2) 82:3 (2010), 663-682. MR 2012d:14026 Zbl 1214.18010

Communicated by David Benson

Received 2013-12-07 Revised 2014-10-13 Accepted 2014-12-06

iyama@math.nagoya-u.ac.jp Graduate School of Mathematics, Nagoya University Chikusa-ku, Nagoya, 464-8602, Japan

peter.jorgensen@ncl.ac.uk

School of Mathematics and Statistics, Newcastle University, Newcastle upon Tyne NE1 7RU, United Kingdom

dongyang2002@googlemail.com Department of Mathematics, Nanjing University, Nanjing, 210093, China 


\section{Algebra \& Number Theory}

msp.org/ant

\section{EDITORS}

MANAGING EDITOR

Bjorn Poonen

Massachusetts Institute of Technology

Cambridge, USA

\author{
EDITORIAL BOARD CHAIR \\ David Eisenbud \\ University of California \\ Berkeley, USA
}

BOARD OF EDITORS

Georgia Benkart

Dave Benson

Richard E. Borcherds

John H. Coates

J-L. Colliot-Thélène

Brian D. Conrad

Hélène Esnault

Hubert Flenner

Edward Frenkel

Andrew Granville

Joseph Gubeladze

Roger Heath-Brown

Craig Huneke

János Kollár

Yuri Manin

Barry Mazur

Philippe Michel

Susan Montgomery
University of Wisconsin, Madison, USA

University of Aberdeen, Scotland

University of California, Berkeley, USA

University of Cambridge, UK

CNRS, Université Paris-Sud, France

University of Michigan, USA

Freie Universität Berlin, Germany

Ruhr-Universität, Germany

University of California, Berkeley, USA

Université de Montréal, Canada

San Francisco State University, USA

Oxford University, UK

University of Virginia, USA

Princeton University, USA

Northwestern University, USA

Harvard University, USA

École Polytechnique Fédérale de Lausanne

University of Southern California, USA
Shigefumi Mori

Raman Parimala

Jonathan Pila

Anand Pillay

Victor Reiner

Peter Sarnak

Joseph H. Silverman

Michael Singer

Vasudevan Srinivas

J. Toby Stafford

Bernd Sturmfels

Richard Taylor

Ravi Vakil

Michel van den Bergh

Marie-France Vignéras

Kei-Ichi Watanabe

Efim Zelmanov

Shou-Wu Zhang
RIMS, Kyoto University, Japan

Emory University, USA

University of Oxford, UK

University of Notre Dame, USA

University of Minnesota, USA

Princeton University, USA

Brown University, USA

North Carolina State University, USA

Tata Inst. of Fund. Research, India

University of Michigan, USA

University of California, Berkeley, USA

Harvard University, USA

Stanford University, USA

Hasselt University, Belgium

Université Paris VII, France

Nihon University, Japan

University of California, San Diego, USA

Princeton University, USA

\section{PRODUCTION}

production@msp.org

Silvio Levy, Scientific Editor

See inside back cover or msp.org/ant for submission instructions.

The subscription price for 2014 is US \$225/year for the electronic version, and $\$ 400 /$ year ( $\$ 55$, if shipping outside the US) for print and electronic. Subscriptions, requests for back issues and changes of subscribers address should be sent to MSP.

Algebra \& Number Theory (ISSN 1944-7833 electronic, 1937-0652 printed) at Mathematical Sciences Publishers, 798 Evans Hall \#3840, c/o University of California, Berkeley, CA 94720-3840 is published continuously online. Periodical rate postage paid at Berkeley, CA 94704, and additional mailing offices.

ANT peer review and production are managed by EditFLOW ${ }^{\circledR}$ from MSP.

\section{PUBLISHED BY}

mathematical sciences publishers

nonprofit scientific publishing

http://msp.org/

(C) 2014 Mathematical Sciences Publishers 


\section{Algebra \& Number Theory}

Volume $8 \quad$ No. $10 \quad 2014$

K3 surfaces and equations for Hilbert modular surfaces

NOAM ELKIES and ABHINAV KUMAR

Intermediate co- $t$-structures, two-term silting objects, $\tau$-tilting modules, and torsion classes

OSAMU IyAma, Peter JøRgensen and Dong YANG

A $p$-adic Eisenstein measure for vector-weight automorphic forms

ELLEN EISCHEN

Explicit points on the Legendre curve III

DOUGLAS ULMER

Explicit Gross-Zagier and Waldspurger formulae

Li CAI, JiE SHU and Ye Tian 